\title{
SPATIAL REPRESENTATION OF USEFUL MINERALS DEPOSITS
}

\author{
Mihai Valentin HERBEI ${ }^{*}$, Roxana Claudia HERBEI ${ }^{2}$, Larisa Ofelia FILIP ${ }^{3}$ \\ ${ }^{1}$ Banat University of Agricultural Sciences and Veterinary Medicine "King Michael I of Romania" Timișoara, \\ Romania,mihai_herbei@yahoo.com \\ ${ }^{2}$ University of Petroșani, Petroșani, Romania, roxanaherbei@yahoo.com \\ ${ }^{3}$ University of Petroșani, Petroșani, Romania, larisafilip@yahoo.com
}

DOI: $10.2478 / \operatorname{minrv-2021-0019}$

\begin{abstract}
Starting from the fact that in the mining industry and in general in the field of underground constructions, the topographic activity, regardless of which of its manifestations, conditions and substantiates the achievement of economic and technological objectives, implicitly resulting in the dependence between topographic precision and qualitative and quantitative performance of mining. The level at which the professional responsibility of the topographic activity, as a whole is engaged in the achievement of some proposed objectives, depends on the framework resulting from the technological complexity of the mining activity in the conditions of its restructuring and efficiency. Spatial representations of useful mineral deposits in the earth's crust, which meet the quality conditions necessary for the design of underground mining works, can be used to choose the most efficient methods of opening, preparation and exploitation of useful mineral deposits. These analyzes are the basis for the realization of underground topographic networks that have a precise connection with the topographic base at the surface. The use of stereographic projection and stereographic networks can be achieved by faithfully representing the tectonics and positioning of deposits of useful mineral matter inside the earth's crust.
\end{abstract}

Keywords: representation, deposits, useful mineral substances, projections

\section{Introduction}

The main objectives of the mining topographic activity are:

- Ensuring the mining activity with the necessary information for the different stages of its development, through the corresponding elaboration of the topographic documentations.

- Applying the concepts regarding the development of the mining activity through the topographic management of the execution of the planned works.

- Realization of a unitary system of representation of the underground geological structures in order to exploit the deposits of useful mineral substance in optimal and efficient conditions.

For this reason, it is necessary to design an efficient and sustainable system, at a higher quality, appropriate level, which allows the optimal fulfillment of the tasks related to these objectives, a decisive role returning in this sense - to optimize underground networks and achieve a unique space reference system.

The spatial system of representation of the underground structures allows the representation of the position of the existing underground works as well as the future development of some underground works based on optimal networks from the point of view of the quality and the way of their accomplishment.

The spatial representation of the deposits of useful mineral substance has a special importance in the positioning of the deposit in the earth's crust using different projections through which to represent as accurately as possible the position of the deposit and the design of mining works necessary for its opening and preparation. [1], [2].

\footnotetext{
* Corresponding author: Mihai Valentin Herbei, Assoc.Prof.PhD.Eng., University of of Agricultural Sciences and Veterinary Medicine "King Michael I of Romania" Timişoara, Romania, contact details (119, Calea Aradului, Timişoara, mihai_herbei@yahoo.com)
} 


\section{Affine projections}

\subsection{The principle of representation}

The construction of some representation in the affine projection [3] is based on the affine transformation of the figures, which consists in the parallel projection of the plane with the given horizontal projection, on another plane after a given design direction. Blueberry projections (fig. 1) are divided into two categories:

- Affine orthogonal projections

- Affine oblique projections

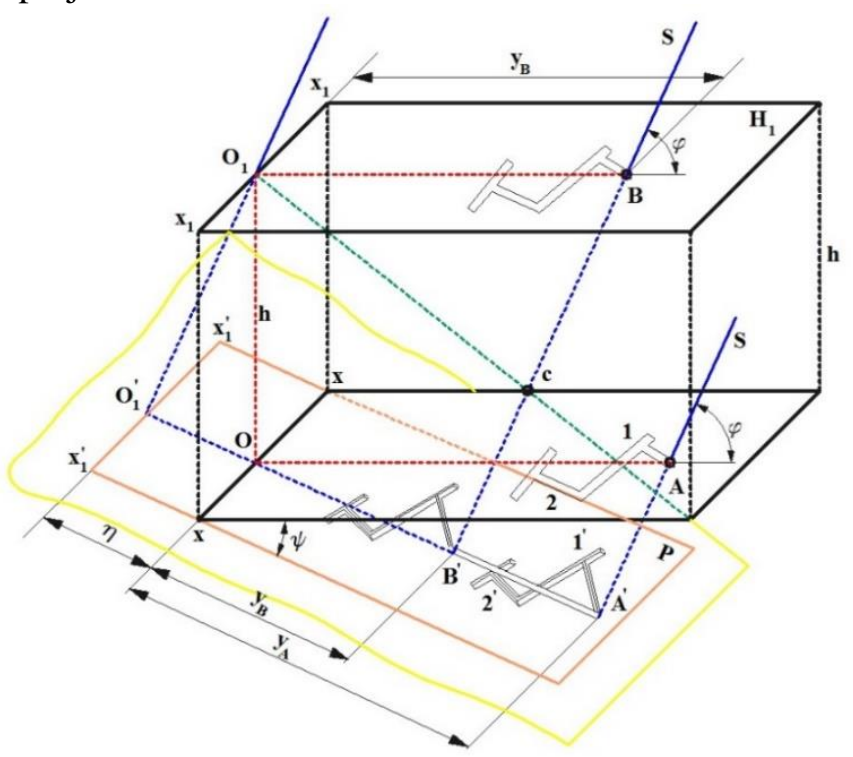

Fig. 1. Affine projection

The properties of affine correspondence are the following [4]:

- Two lines that are in the affine correspondence may intersect at a point on the affine axis, or are parallel to this axis.

- The lines that in the initial plane were parallel to it do not deform.

- The ratio of the distances of a certain pair of points, for given design conditions, is a constant quantity and is called the transformation coefficient $\mathrm{q}$.

$$
\begin{aligned}
& q=\frac{A^{\prime} O}{A O}=\sin \varphi \\
& A O=A O \sin \varphi \\
& I_{a}=\frac{A^{\prime} O}{A O}=\sin \varphi
\end{aligned}
$$

The coordinates of the affine points, for example of points B and B1, are found in the following relation to the affine axis.

$$
\begin{aligned}
& O_{1} C=O_{1} B \sin \varphi \\
& O_{1} C=O_{1}^{\prime} O+O B^{\prime}=\eta+y_{b} \\
& q_{1} B=Y_{b}
\end{aligned}
$$

From the triangle $O O_{1} O_{1}^{\prime}$ :

$$
\begin{aligned}
& O_{1}^{\prime} O=H \cos \varphi \\
& \eta=h \cos \varphi \\
& I_{b}=Y_{b} \sin \varphi-h \cos \varphi
\end{aligned}
$$

With this relation the affine ordinates $\mathrm{y} 1$ are determined according to the ordinates $\mathrm{Y}$ from the initial plane Ho, the projection angle $\varphi$ and the level difference $h$ between the given point and the affine axis. From the relation it results that for another level $\mathrm{H}_{1}$ all the ordinates of the points included in this plane decrease with a constant magnitude $\eta$.

\subsection{Methods for making affine representations}

For the construction of the affine projection of a certain complex of details - mining works at different levels - the horizontal projection with the grid of the coordinate system against which the respective points were represented is considered [5]. 
The graphic procedure (fig. 2) consists in choosing the affine axis XX to which all the characteristic points of the respective objective are projected orthogonally. Separately, the actual affine representation of the given situation is constructed. Draw the direction of the blueberry axis XX for the first level, usually the horizon with greater depth.

Mark all the projected points from the respective level in the direction of this axis. By drawing the blue ordinates, the points that join and from which the respective detail represented in blue are obtained are obtained. Next, the affine axis is drawn for the next level with the projections of the characteristic points on the respective horizon (fig. 3).

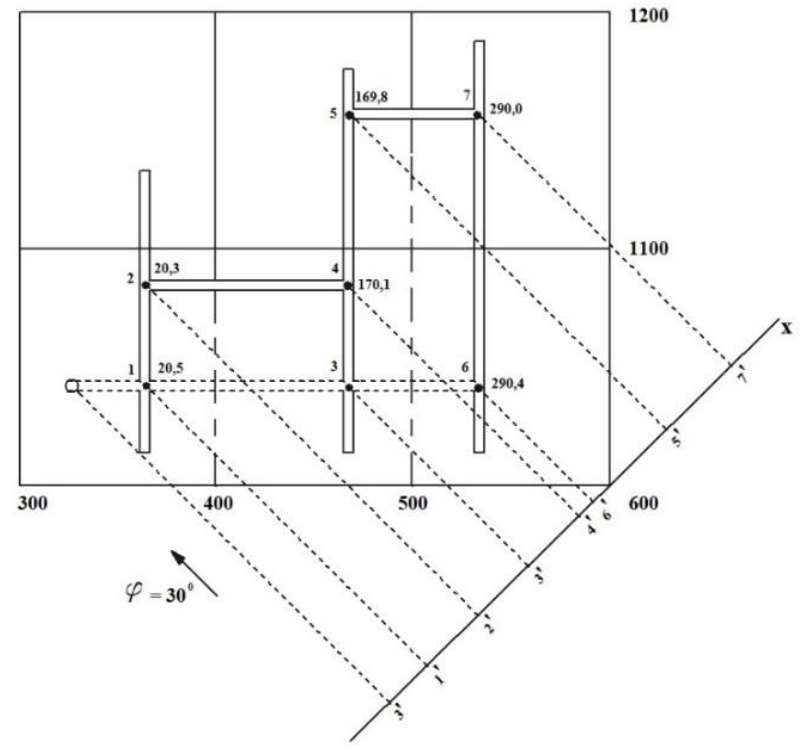

Fig. 2. Graphic procedure
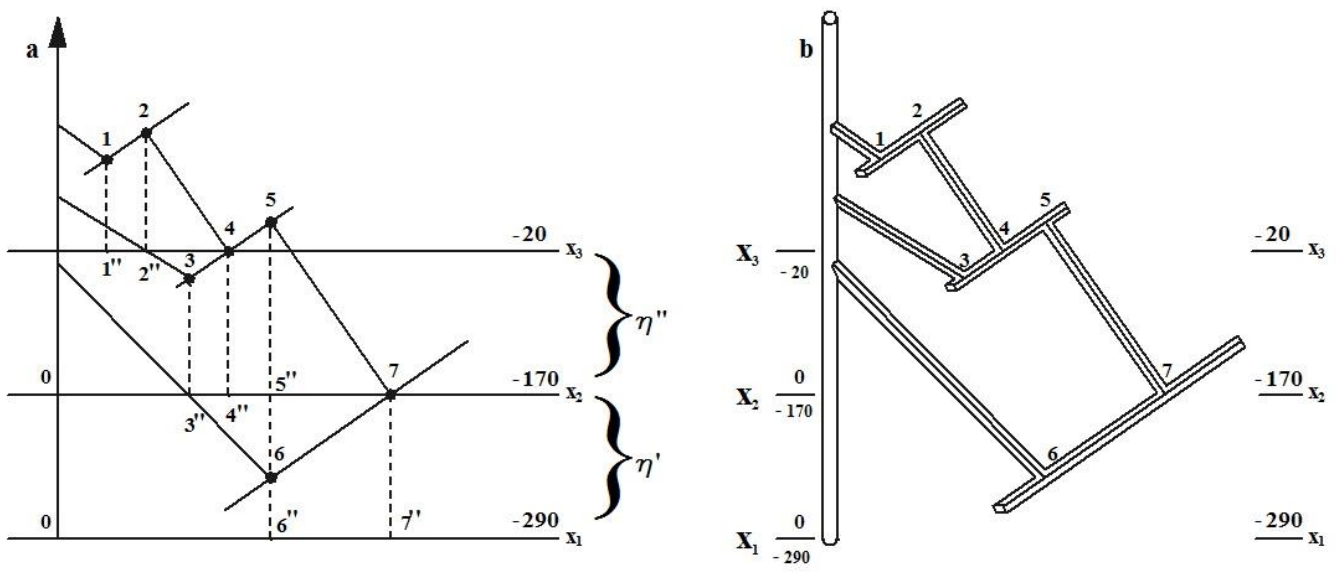

Fig. 3. Graphic processes - Representation in space of details

The analytical process consists in calculating all the affine coordinates of the given points in a horizontal projection. The calculation is made after the $\mathrm{X}$ and $\mathrm{Y}$ axes have been drawn on the initial plane, according to which the values of the coordinates of the points are appreciated. The affine representation itself is also done separately as in the graphic method [6].

\section{Axonometric projections}

Axonometric projections [7] are a particular case of parallel projections. The principle of axonometric projection consists in the fact that the object represented in a system of three rectangular axes is projected on another projection plane, together with the initial system of coordinate axes itself (fig. 4).

Depending on the angle between the design direction and the projection plane, there are two categories of axonometric projections:

- Oblique axonometric projections.

- Orthogonal axonometric projections. 


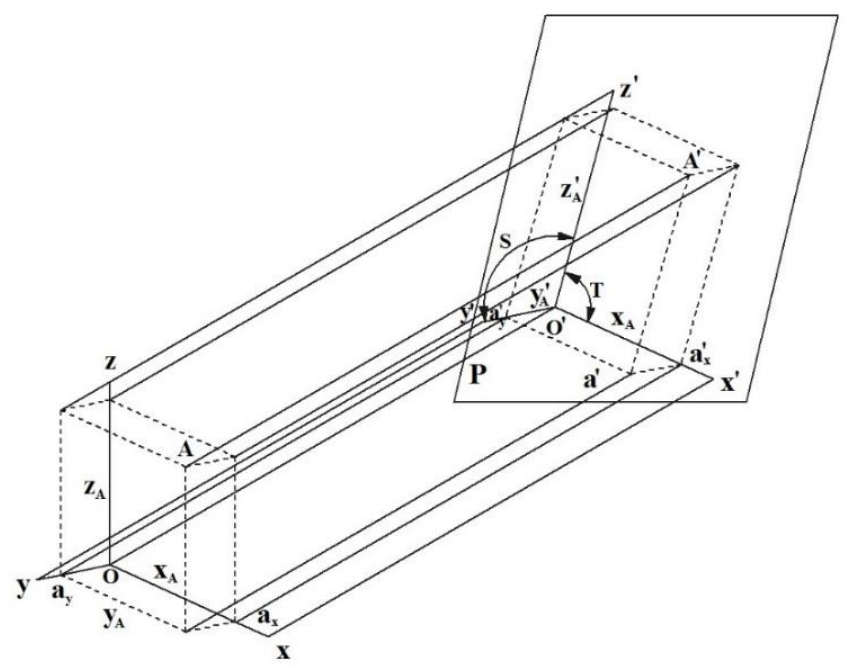

Fig. 4. Axonometric projection

Figure 4 shows the parallel design of a point $\mathrm{A}$ in space, together with the axes of the $\mathrm{O}_{\mathrm{XYZ}}$ system on the axonometric plane $\mathrm{P}$. On the plane $\mathrm{P}$ is obtained the projection of the point $\mathrm{A}$ as well as the directions of the axonometric axes. If the equal segments Oax, Oay, Oaz are considered on each axis of the initial $\mathrm{O}_{\mathrm{XYZ}}$ system, they will be projected on the corresponding directions of the axonometric axes.

The ratio of the axonometric projections to the real sizes of the considered segments represents the deformation coefficient for the axes and it is noted:

$$
\begin{aligned}
& p=X^{\prime} / X \\
& q=Y^{\prime} / Y \\
& r=Z^{\prime} / Z \\
& p^{2}+q^{2}+r^{2}=2 \\
& S=90^{\circ}+\frac{\alpha}{2} \\
& T=90^{\circ}+\frac{\gamma}{2}
\end{aligned}
$$

where: $\mathrm{S}$ - the angle between the axes $\mathrm{O}^{\prime} \mathrm{Z}^{\prime}$ and $\mathrm{O}^{\prime} \mathrm{Y}^{\prime}$, $\mathrm{T}$ - the angle between the axes $\mathrm{O}^{\prime} \mathrm{Z}^{\prime}$ and $\mathrm{O}^{\prime} \mathrm{X}^{\prime}$

$$
\begin{aligned}
& \cos \alpha=\frac{\left(q^{2}\right)^{2}+\left(r^{2}\right)^{2}+\left(p^{2}\right)^{2}}{2 q^{2} r^{2}} \\
& \cos \gamma=\frac{\left(q^{2}\right)^{2}+\left(r^{2}\right)^{2}-\left(q^{2}\right)^{2}}{2 q^{2} r^{2}}
\end{aligned}
$$

If $\mathrm{p}=\mathrm{q}=\mathrm{r}$ isometric projection. In this case the axonometric axis $\mathrm{OZ}$ has a vertical position and makes the angle of 1200 with the axes $\mathrm{O}_{\mathrm{X}}$ and $\mathrm{O}_{\mathrm{Y}}$. The deformations on all three axes are 0.816 , for the simplification of the construction they can be considered equal to 1 .

If $\mathrm{p} \neq \mathrm{q}=\mathrm{r}$ is the dimetric projection. The axonometric axis $\mathrm{OZ}$ has a vertical position and the axis $\mathrm{O}_{\mathrm{Y}}$ is taken horizontally. The angle between $\mathrm{O}_{\mathrm{X}}$ and $\mathrm{O}_{\mathrm{Y}}$ is equal to 1350. The deformation coefficients for the $\mathrm{O}_{Z}$ and $\mathrm{O}_{\mathrm{Y}}$ axes are considered equal, and for the $\mathrm{OX}$ axis the value is considered twice smaller. Thus the values of the coefficients are considered: $\mathrm{p}=0.5 ; \mathrm{q}=\mathrm{r}=1$.

If $\mathrm{p} \neq \mathrm{q} \neq \mathrm{r}$ the trimetric projection. If the coefficients have different values, the axonometric axis is directed vertically, and the axes $\mathrm{O}_{\mathrm{X}}$ and $\mathrm{O}_{\mathrm{Y}}$ form with the horizontal the angle of 100 and 450 respectively. The values currently used for the deformation coefficients are: $\mathrm{p}=0.9 ; \mathrm{q}=0.5 ; \mathrm{r}=1$.

In order to build an axonometric projection, it is necessary to represent the respective details in a dimensioned plan.

\section{Vector projections}

One of the adequate methods for the representation of mining works and geological formations is the vector projection (fig. 5). Vector representations are used in the field of mining technology, especially in solving mining breakthrough problems due to the simple, clear and easy to use construction.

The vector projection of a certain segment $\mathrm{AB}$ from space (fig. 5) is made by carrying through the points $\mathrm{A}$ and $\mathrm{B}$ parallel vectors in a certain direction, but of lengths proportional to the dimensions of the respective points are obtained in the horizontal projection plane $\mathrm{H}$ two directions $\mathrm{ab}$ and $\mathrm{a}$ ' $\mathrm{b}$ ' which represents the vector projection of the segment $A B$. 


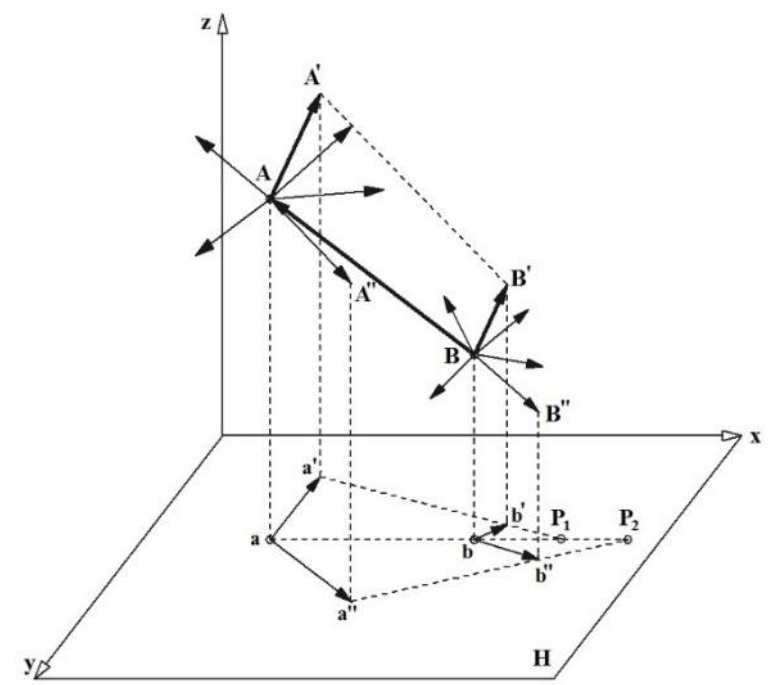

Fig. 5. Vector projection

According to the reciprocal position of the segments ab and a'b ', assessments can be made on the position of the line in space with respect to the reference plane $\mathrm{H}$. The point $\mathrm{P} 1$ of intersection of the two directions is called the center of resemblance and is located in the reference plane $\mathrm{H}$. between the points in space and the plane is determined by the size of the respective vectors and their direction indicates the position of the points in space with respect to the reference plane (above or below it).

The line parallel to the reference plane is represented vector by two parallel directions, and the vectors considered at any point of it will be equal in length.

The line perpendicular to the reference plane is projected vector after two vectors starting from the same point and confusing in one direction.

A straight line included in the vertical plane perpendicular to the reference plane $\mathrm{H}$ is projected vector also after two vectors confused in one direction but starting from two different points.

\section{Central projection}

The problems often encountered in mining geometry regarding the performance of exploration and exploitation of deposits of useful mineral substance, are construction problems in which it is required to determine the values of angles and directions of both lines and planes, problems presented and analyzed in the previous paragraphs.

In solving these problems, the central projection has a special importance. The characteristic of this projection is the fact that all the directions and planes given in space are transported parallel to themselves in a point around which a sphere is described. The sphere will intersect with the given planes and lines in the form of circles and points. These intersections are carried on a plane by a beam of projection beams that allow easy determination of the angles sought between the given directions and planes. Depending on the character of the reciprocal position of the projection center (observation point) and of the projection plane with respect to the sphere are used:

- Linear projection

- Stereographic projection

\subsection{Linear projection}

In the case of linear projection (fig. 6) the projection center is located in the center of the sphere and the projection plane is tangent to the sphere at point $\mathrm{Z}$. The projections of directions and planes are obtained by points and lines. The distance between them and the fixed point $\mathrm{Z}$ depends on the angles of inclination of the directions and planes with respect to the projection plane which is determined by the relation:

$$
\overline{Z_{m}}=\operatorname{Rctg} \delta=\operatorname{Rtg}\left(90^{\circ}-\delta\right)
$$

The disadvantage of this projection is that as the angle decreases, the projection of directions and planes deviates from the main point $\mathrm{Z}$. 


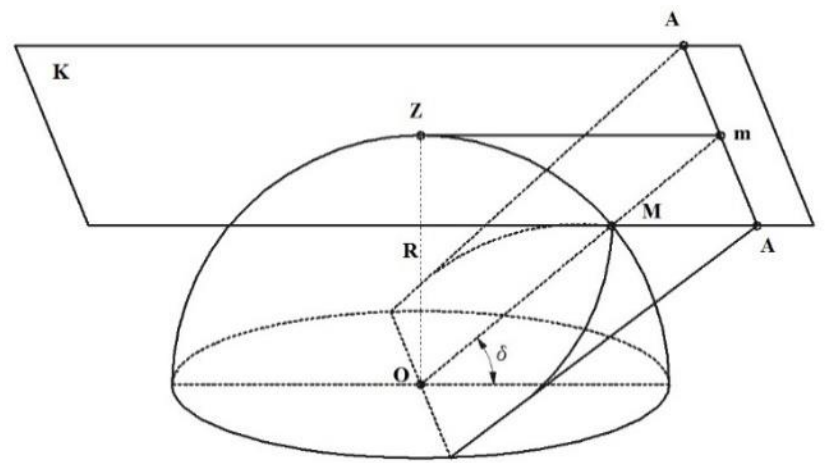

Fig. 6. Linear projection

\subsection{Stereographic projection}

In the case of stereographic projection, the center of projection is considered to be located somewhere on the surface of the sphere and the geometric elements (axes, planes, etc.) intersect the reference sphere passing through its center. All the details of the problems are projected on the sphere according to a coordinate system made up of a set of orthogonal arcs analogous to the meridians and parallels of the globe [8], [9].

The properties and advantages of stereographic projection are:

Inside the basic circle (the section of the sphere with the projection plane) the two hemispheres can be fully represented.

Stereographic projection is a compliant projection. The size of the angles between the arcs of the circles representing the planes in stereographic projection is equal to the actual size of the angles between the planes.

The circumferences of large and small circles are represented in the stereographic projection by circles (arcs of a circle) and in particular cases (when representing vertical planes) by lines. The projection of the plan is a circle.

The projection center is located on the surface of the sphere at the end of the diameter $Z$ - $Z$ ' perpendicular to the projection plane (fig. 7). The projection plane $\mathrm{K}$ passes through the center of the sphere, which has the advantage that the directions and planes with small values of the angles of inclination are easily and accurately represented within the drawing. The projections of directions and planes are represented in the form of circles, lines and points. The direction $\mathrm{BC}$ will be represented in the stereographic projection by the segment bc. All directions pass through the center of the sphere (point $\mathrm{O}$ ), the direction $\mathrm{BC}$ will be perfectly determined by the position of a point $\mathrm{B}$. The plane passing through the direction $\mathrm{BC}$ gives on the sphere, the large circle CEBD whose stereographic projection on the plane $\mathrm{K}$ is represented by the circle $\mathrm{CEBD}$. The angles of inclination to the horizontal projection plane of the plane CEBD and the direction $\mathrm{BC}$ are determined by the segment in the expression:

$$
\overline{O b}=\operatorname{Rtg}\left(\frac{90-\delta}{2}\right)
$$

According to the position of the projection plane with respect to the sphere, the following stereographic projections are used to solve the tectonic disturbances regarding the tectonic disturbances, geological anomalies, rock fissures, mining penetrations, determination of the thickness and inclination of the deposits and determination of their thickness and shape:

- oblique stereographic projection;

- polar stereographic projection;

- meridian (equatorial) stereographic projection. refer to:

The analyzes presented regarding the geometric problems of the deposits of useful mineral substance that

- crystallographic problems;

- petrographic problems;

- mining topography problems.

These problems can be solved using stereographic networks which can be polar stereographic projection, when the projection plane is superimposed on the equatorial plane of the sphere and the observation point corresponds to the nadir or it can be stereographic projection with meridian plane, when the observation point is in an any point on the equator and the projection plane in a meridian plane located at 900 observation points. 


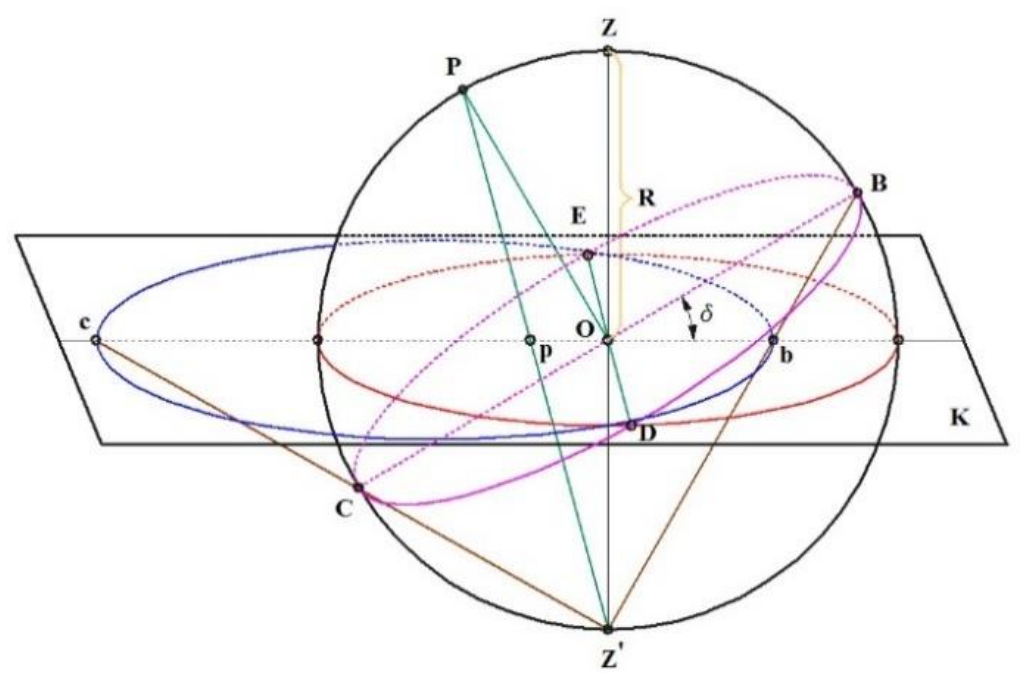

Fig. 7. Stereographic projection

\section{Use of polar stereographic networks}

Polar stereographic networks accurately represent the angular position elements of the axes and planes or the angles between them, so the angular ratios of the elements considered. Both modes of representation significantly distort the surfaces, so the ratio of lengths.

For the study of rock massifs in general of the tectonics of the deposits according to the value of the slope angle of the fissure planes, the stereographic polar projections or the orthographic polar projections are used. Thus, if in the researched field most of the fissures have large slope angles (over 450) of the respective planes, and then it is recommended to use orthographic polar projections.

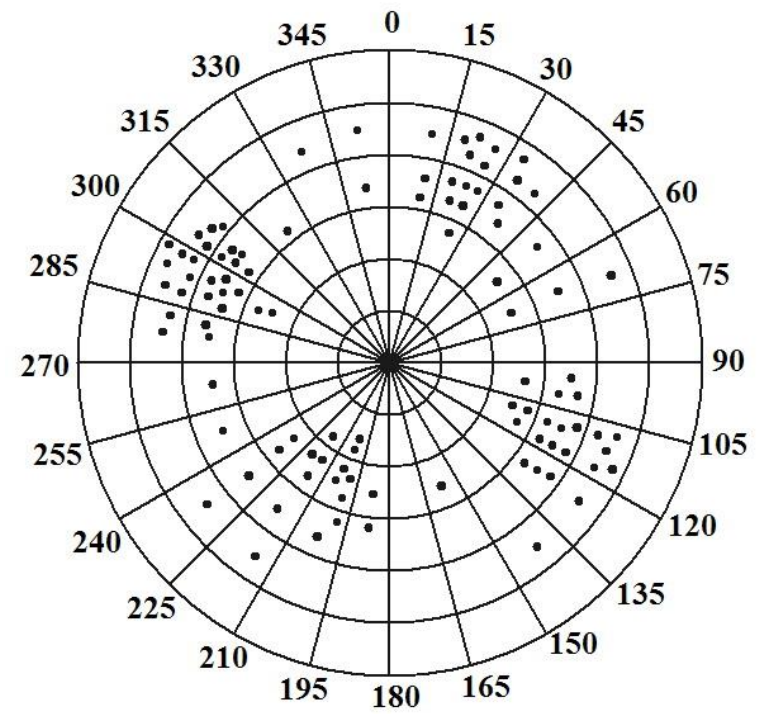

a

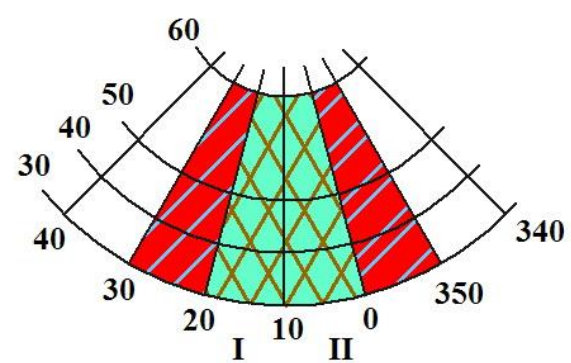

b

Fig. 8. Transcript of studying rock fissure

By using stereographic networks and drawing up stereograms (fig. 8), the fissuring of rocks in a certain massif can be analyzed using a statistical processing of the data obtained by stereogram. Thus, it is considered an abacus bordered by rays and parallels at intervals of 100,200, which moves on the surface of the stereogram. In each position of the abacus is determined the number of points or fissures, which are contained within it, noting the figure at the center of the position. Thus, a series of values are obtained that can be expressed as a percentage, and by interpolation the isolines of the fissure concentration in the studied area are drawn.

The processing of the individual fissure data in order to trace the isolates of the concentrations is done with the help of an abacus. The choice of the size of the abacus surface depends on the degree of detail with which the respective insulations must be built. Fissure concentration isolines (Fig. 9) are drawn within 3\%. 


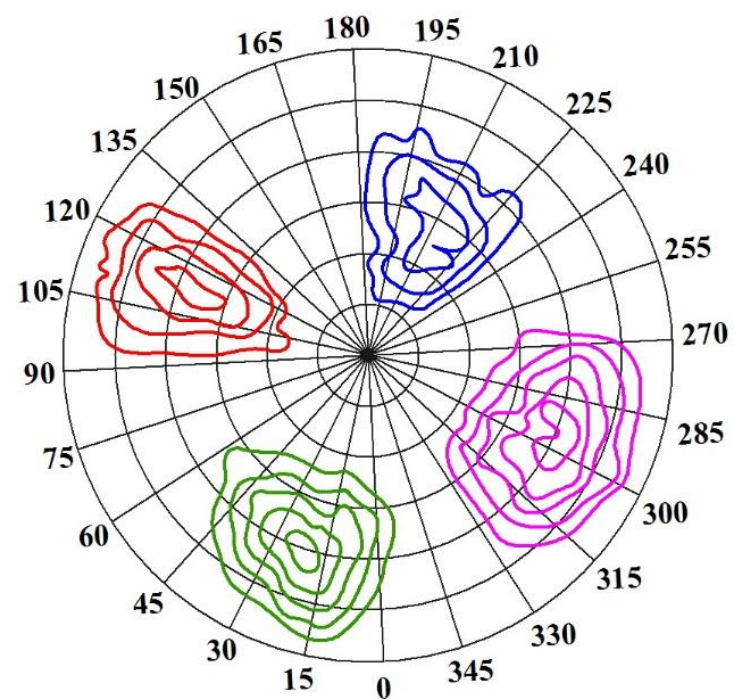

Fig. 9 Fissure concentration

\section{Graphical representation of the discontinuities of a deposit}

The structural elements of a deposit measured in the field are statistically processed in order to establish a correlation. between the overall or detailed structure of the deposit (in the case of coal deposits) and the degree of fissuring of the coal, as well as the correlation between these discontinuities and the tectonic disturbances of the geological structures. For the design and statistical processing of the sets of micro tectonic elements, several methods and methods are used, which are chosen according to the purpose pursued. Usually the statistical design and processing of the sets of micro tectonic elements is performed with the help of structural diagrams or frequency histograms.

The frequency diagram is used to represent the directions or inclinations of the discontinuities. The diagram is divided radially into circle sectors of 100 as well as according to a number of concentric circles chosen according to the number of measurements.

After the field measurements, a table is drawn up in which the fissures are grouped with the same or very close directions $(\approx 50)$, thus obtaining several groups of measurements. The direction of the group of fissures thus obtained is projected in a semicircle in the sector between the corresponding radii, and the number of measurements of the group is taken on the radius of the circle starting from the center of the semicircle. To obtain the length of the radius segment corresponding to a measurement, divide the radius of the circle by the largest number of measurements found in a group. The length of the circle sector corresponding to a group of measurements will be the greater the number of measurements. The group with the most measurements will coincide with the length of the radius of the semicircle (fig. 10).

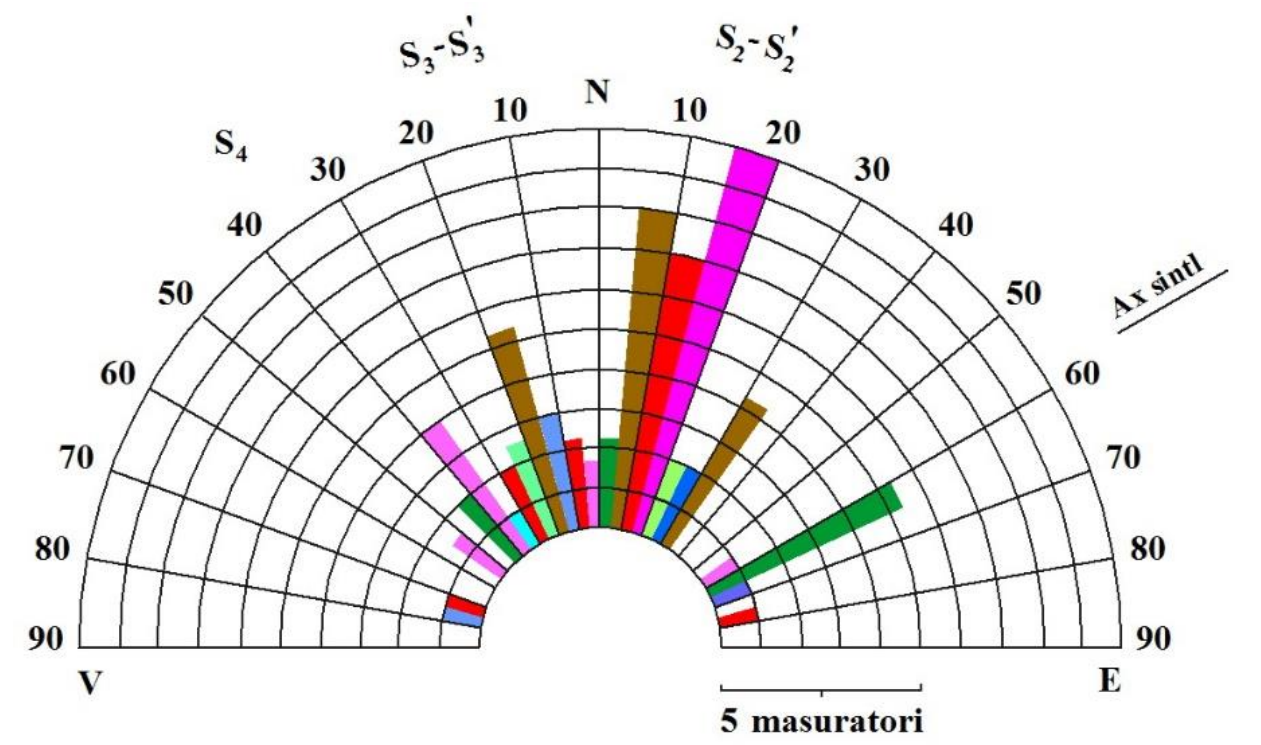

Fig. 10. Fissure direction frequency diagram 
The method of representation in two-dimensional diagrams is incomparably superior to one-dimensional representation. Two-dimensional representation is a spherical projection in which the stereographic projection network is commonly used. The network keeps the spherical surfaces in projection, modifying them only in a very small proportion.

The design of micro tectonic elements in the stereographic network is done, in case of a large number of measurements, by statistical processing using specific software.

\section{Conclusions}

From the analysis of the spatial configuration of deposits, tectonic accidents and mining works for the optimal realization of mining works for the extraction of useful mineral substances in conditions of maximum quality, efficiency and safety we will have to analyze the spatial representation systems of geological and underground mining structures.

Following this study it can be concluded that the spatial representations of the deposits of useful mineral substance in the earth's crust, which meet the quality conditions necessary for the design of underground mining works, can be used to choose the most efficient methods of opening, preparation and exploitation of deposits of useful mineral substance. These analyzes are the basis for the realization of underground topographic networks that have a precise connection with the topographic base at the surface.

From the presented analysis it can be concluded that the use of stereographic projection and stereographic networks can be achieved by faithfully representing the tectonics and positioning of the deposits of useful mineral substance inside the earth's crust. Based on the presented analyzes, the further development of the skeleton of the underground mining works will be established in order to efficiently extract the useful mineral substance.

\section{References}

[1] Domide E., Filimon R., 1962

Mining topography, Technical Publishing House, Bucharest

[2] Marian, D.P., Onica I., 2021

Finite element modelling of the stability of underground mining excavations al old mines - Slanic Salt Mine, Mining Revue, 27(1), 12-23

[3] Ozeki, K., 2016

Affine Projection Algorithm. In: Theory of Affine Projection Algorithms for Adaptive Filtering. Mathematics for Industry, Springer, Tokyo. https://doi.org/10.1007/978-4-431-55738-8_3

[4] Ozeki, K., Umeda, T., 1984

An adaptive filtering algorithm using an orthogonal projection to an affine subspace and its properties. Electronics and Communications in Japan (Part I: Communications), 67(5), 19-27.

[5] Sabatin, R., 1971

Geometry of deposits, IM Petrosani

[6] Dima N., Pădure I., Herbei O., 1996

Mining topography, Corvin Publishing House Deva

[7] Herbei, R., 2011

Ph.D Thesis Petrosani

[8] Filip, L., 2021

Increasing the accuracy of the orientation of underground topographic works, Mining Revue, 27(1) 66-69

[9] Ortelecan M. Palamariu M., Jurca T., 1999

Tracing of mining works, Infomin Publishing House Deva

This article is an open access article distributed under the Creative Commons BY SA 4.0 license. Authors retain all copyrights and agree to the terms of the above-mentioned CC BY SA 4.0 license. 